\title{
Use of linear regression models to determine influence factors on the concentration levels of radon in occupied houses
}

\author{
Jonas Buermeyer \\ Institute for Medical Physics and Radiation Protection, THM University of Applied Sciences Mittelhessen, \\ Wiesenstr. 14, Giessen, 35390/Hesse, Germany \\ Jonas.Buermeyer@kmub.thm.de \\ Matthias Gundlach \\ Department of Math, Natural Science and Information Technology, THM University of Applied Sciences \\ Mittelhessen, Wiesenstr. 14, Giessen, 35390/Hesse, Germany \\ Matthias.Gundlach@mni.thm.de \\ Anna-Lisa Grund \\ Institute for Medical Physics and Radiation Protection, THM University of Applied Sciences Mittelhessen, \\ Wiesenstr. 14, Giessen, 35390/Hesse, Germany \\ Anna-Lisa.Grund@mni.thm.de \\ Volker Grimm \\ Institute for Medical Physics and Radiation Protection, THM University of Applied Sciences Mittelhessen, \\ Wiesenstr. 14, Giessen, 35390/Hesse, Germany \\ Volker.Grimm@mni.thm.de \\ Alexander Spizyn \\ Institute for Medical Physics and Radiation Protection, THM University of Applied Sciences Mittelhessen, \\ Wiesenstr. 14, Giessen, 35390/Hesse, Germany \\ Alexander.Spizyn@kmub.thm.de \\ Joachim Breckow \\ Institute for Medical Physics and Radiation Protection, THM University of Applied Sciences Mittelhessen, \\ Wiesenstr. 14, Giessen, 35390/Hesse, Germany \\ Joachim.Breckow@mni.thm.de \\ Published 1 September 2016
}

This work is part of the analysis of the effects of constructional energy-saving measures to radon concentration levels in dwellings performed on behalf of the German Federal Office for Radiation Protection. In parallel to radon measurements for five buildings, both meteorological data outside the buildings and the indoor climate factors were recorded. In order to access effects of inhabited buildings, the amount of carbon dioxide $\left(\mathrm{CO}_{2}\right)$ was measured. For a statistical linear regression model, the data of one object was chosen as an example. Three dummy variables were extracted

This is an Open Access article published by World Scientific Publishing Company. It is distributed under the terms of the Creative Commons Attribution 3.0 (CC-BY) License. Further distribution of this work is permitted, provided the original work is properly cited. 
from the process of the $\mathrm{CO}_{2}$ concentration to provide information on the usage and ventilation of the room. The analysis revealed a highly autoregressive model for the radon concentration with additional influence by the natural environmental factors. The autoregression implies a strong dependency on a radon source since it reflects a backward dependency in time. At this point of the investigation, it cannot be determined whether the influence by outside factors affects the source of radon or the habitant's ventilation behavior resulting in variation of the occurring concentration levels. In any case, the regression analysis might provide further information that would help to distinguish these effects. In the next step, the influence factors will be weighted according to their impact on the concentration levels. This might lead to a model that enables the prediction of radon concentration levels based on the measurement of $\mathrm{CO}_{2}$ in combination with environmental parameters, as well as the development of advices for ventilation.

Keywords: Statistics; Regression Analysis; Radiation Protection; Radon.

\section{Introduction}

Since it is common knowledge that radon is responsible for about $40 \%$ of the natural radioactive exposure, it is important to monitor its concentration in the air, especially in occupied buildings. In order to achieve the climate goal of reducing the concentration of carbon dioxide $\left(\mathrm{CO}_{2}\right)$, the German government passed a law regarding an increase of the energy efficiency of buildings. ${ }^{1}$ This applies especially for new buildings but also comes into account when old buildings undergo a renovation. It is assumed that due to the usage of special insulation material to decrease heating costs, as well as with improved sealing of doors and windows, the average radon concentration in these buildings increases. This might result in an elevated radon exposure for the inhabitants but should not be higher than $300 \mathrm{bq} / \mathrm{m}^{3}$ according to the EURATOM basic safety standards, which become German federal law on February $18^{\text {th }} 2018 .^{2}$

The German Federal Office of Radiation Protection funded a project (BfS $3611 \mathrm{~S}$ 10016 Los 2) that especially covers the aforementioned aspects concerning renovated buildings. Its intention was the identification of effects of constructional energy-saving measures on radon concentration levels in dwellings. Therefore, the radon concentration was monitored in different objects. In parallel, both meteorological data outside the buildings as well as the indoor climate factors were recorded. In order to assess the influences of residents within buildings, the amount of $\mathrm{CO}_{2}$ and its daily progress was recorded. Besides a basic graphical analysis of the data, a statistical approach was taken. In the first step, correlation coefficients were investigated. Slight dependencies could be detected, but they did not lead to significant correlation coefficients. Therefore, further investigations focused on linear regression models in order to quantify possible influences of climate parameters on the radon concentration. Such results might even allow forecasts on radon levels and provide a basis for advice on ventilation behavior in buildings. For this investigation, a sample set of one house was used. Measurements were taken in the basement, living room and entrance hall on the ground floor and two bedrooms in the upper floor. This results in five data sets consisting of the radon levels, the $\mathrm{CO}_{2}$ concentration, temperature, air pressure and humidity, and the outside weather parameters. From these five data sets, five statistical regression models were calculated. 


\section{Method}

For the computation of the linear regression models, the statistical open source software $\mathrm{R}$, developed by the R-project community, was used. ${ }^{3}$ It calculates the regression coefficients using the least squares method. Special care was taken of the model's residuals, which must fulfill three conditions: ${ }^{5}$

- The residuals must be independent from each other

- Residuals must be distributed normally

- Residuals need to be homoscedastic

To meet these, the model had to undergo transformations and needed further corrections.

The dependent variable of the model is the measured radon level of each location in an object. The variables estimated to influence the radon level are represented by climate and meteorological parameters. It was assumed that the gradients of these factors are responsible for variations in the radon levels. The gradients are calculated from the temperature, humidity, and air pressure outside and inside the building. Furthermore, the meteorological parameters rain and wind were also expected to have an impact on the measured radon concentration. Along with the aforementioned factors, the occupant's behavior had to be represented in the model because it is known that the air exchange rate is the most important factor for the radon saturation in buildings. The ability to consider the ventilation behavior is achieved by extracting three dummy variables ${ }^{4}$ from the progress of the $\mathrm{CO}_{2}$ concentration. Dummy variables are dichotomous, meaning they can only express two values ' 0 ' or ' 1 ' if defined criteria are met. The first variable represents the usage of a room by occupants. The residents are assumed to be the only source of $\mathrm{CO}_{2}$ within the building. This allows for marking of occupants' presence by analyzing the $\mathrm{CO}_{2}$ data for times which show rising concentration levels. These periods are set as ' 1 ' in the dummy variable. Times of falling $\mathrm{CO}_{2}$ levels indicate a moderate ventilation of the room. This is marked by a second dummy variable. The third variable can be interpreted as a marker variable for high rates in air exchange. This means a difference in the $\mathrm{CO}_{2}$ concentration of at least ten percent within ten minutes is marked ' 1 ' within the dummy variable whereas the rest is marked ' 0 '.

After the data preparation and definition of the basic structure of the model (radon as the dependent variable, whereas the other parameters build the independent variables), an initial simple linear model was generated. The evaluation made obvious that the residuals did not match the needs described previously. They showed a log-normal distribution rather than a normal distribution. As a conclusion, the dependent variable (radon) was transformed logarithmically to achieve normality in the distribution of the residuals with respect to the definition of the residuals.

In comparison to the non-transformed model shown in Fig. 1, the positive effect of the logarithmic transformation is shown in detail in Fig. 2. It illustrates that there are still minor differences of the normal distribution according to the minor differences in the residuals, resulting in a right positive shift. The Q-Q Plot makes this even more clear. 
Since radioactive decay is a statistical process, this effect might be the result of a lack of sensitivity of the measuring device for low levels of radon resulting in high variances in the acquired data. Due to this effect, the regression model tends to overestimate the radon concentration especially for low levels of radon. The minor differences in the distribution can be neglected to fulfill the need of normally distributed residuals particularly for models with an average radon concentration of $50 \mathrm{bq} / \mathrm{m}^{3}$.
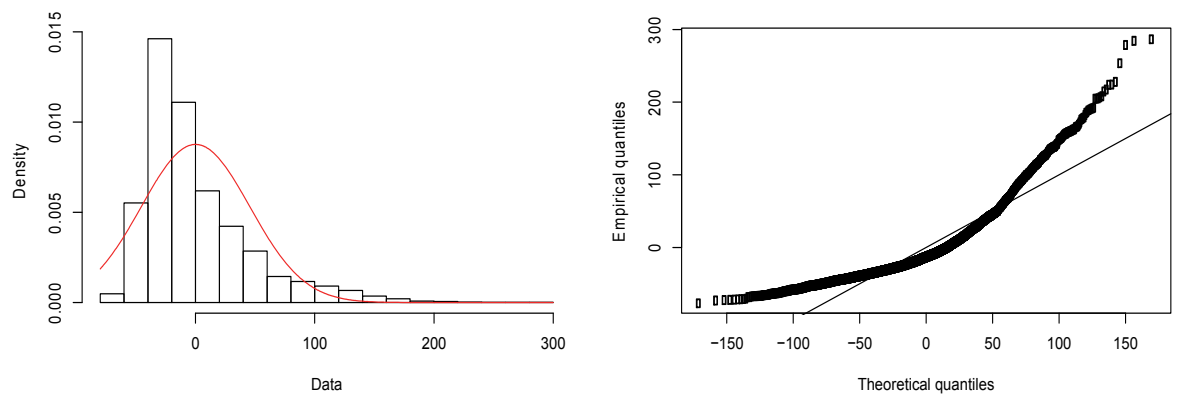

Fig. 1. Residuals of untransformed regression model.
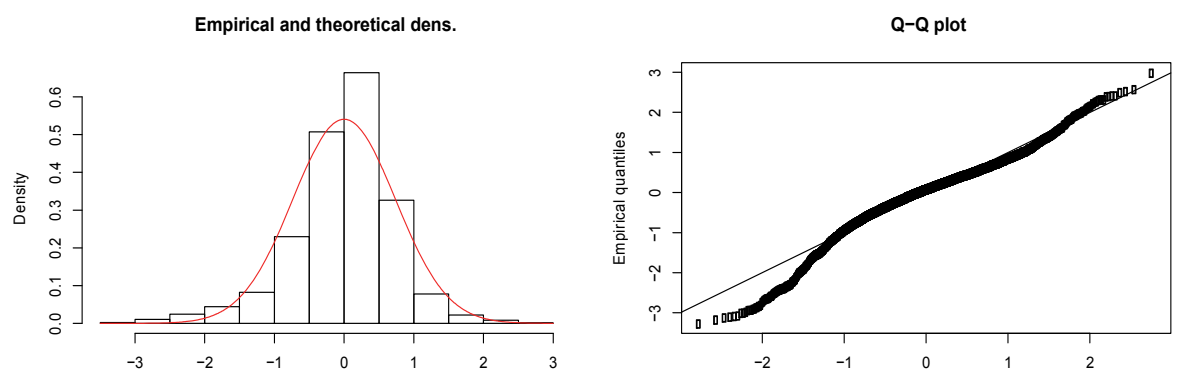

Fig. 2. Residuals of log-transformed regression model.

The next step in the investigation is focused on the determination of independent residuals of the log-transformed model. This resulted in a strong autocorrelation of the residuals. It was assumed that this is caused by the highly autoregressive behavior of the radon concentration. An immediate analysis confirmed this presumption: the radon concentration could be displayed as an autoregressive model of first order. This knowledge provided information sufficient to resolve the problem of dependent residuals. The autoregressive behavior of the gas concentration was considered in the model by adding time-shifted variables of the radon levels.

The effect of this change on the model is presented in Fig. 3. The left part of the figure shows the autocorrelation of the residuals. The high correlation value indicates a strong dependence of the residuals among themselves, whereas the right side states the 
resolved problem with no sign of autocorrelation. At this point it has to be pointed out that the value at lag 0 is always 1 .

\section{Autocorrelation of Residuals}
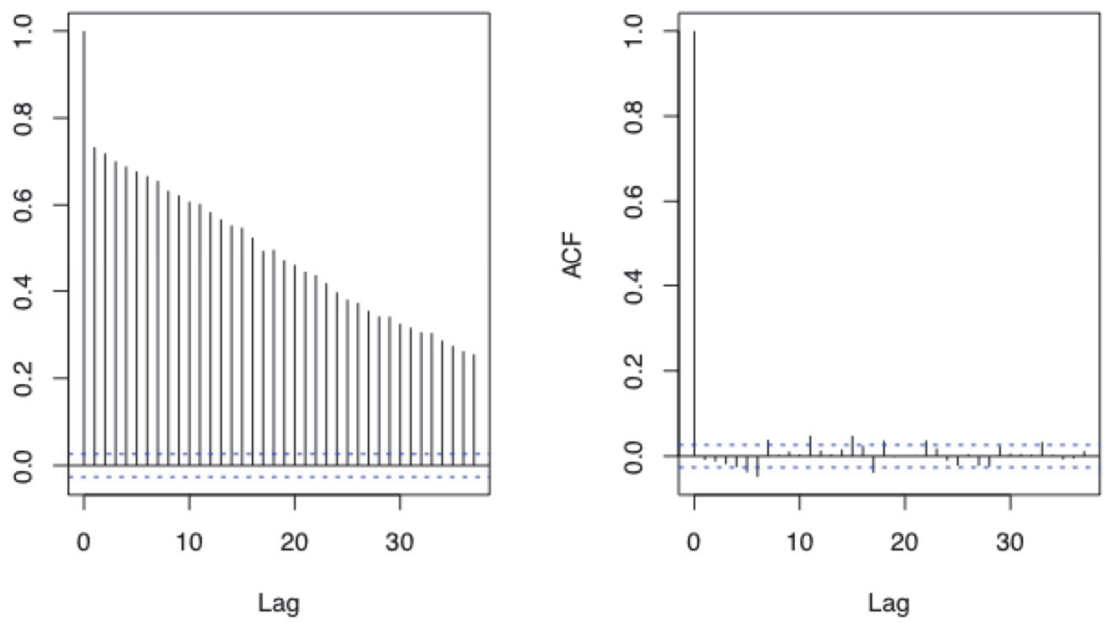

Fig. 3. Autocorrelation of Residuals, left: Residuals of model without respect to autocorrelation, right: Residuals with consideration to autocorrelation.

The final condition for applying the least squares method for estimating regression coefficients concerns the variance of the residuals. The variance of the residuals should be homoscedastic, which means the variance should be zero or at least be assumed as constant within a fixed interval. Figure 4 implies that the residuals show a heteroscedastic behavior. The variance decreases with increasing values for the radon concentration. So the requirement of homoscedasticity is not fulfilled. Therefore, weighting factors for the residuals are estimated by using the "White estimate" or "White error". ${ }^{3}$ This results in a reinterpretation of the estimates gained in the model and corrects for homoscedasticity. The model built on the basis of the mentioned transformations and corrections can now be built for each location of the object where measurement took place. The model initially consisted of many variables with no significance (significance tests are included in R-modeling function). Non-significant variables were removed recursively until the model consisted only of significant variables. Significant variables that occurred several times were analyzed with respect to their directed influence. Only those input variables were left in the model, which showed a consistent effect on the radon concentration. 


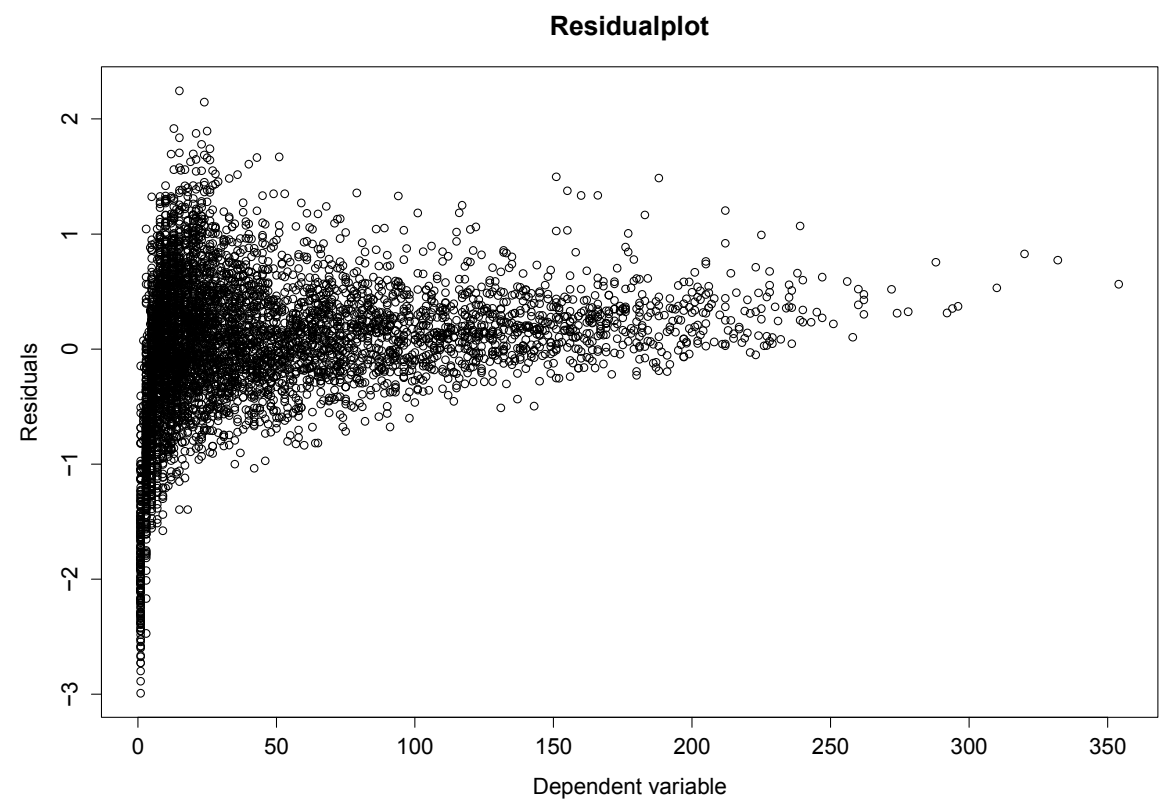

Fig. 4. Example Residualplot.

\section{Results}

A sample output of a statistical model is shown in Fig. 5. The five statistical models created from the measured data show some individual behavior as well as some similarities: all models show a similar structure in the autoregressive terms particularly with respect to the time lags. This underlines the influence of the radon source on the radon concentration and the transportation of radon by the airflow.

The variables concerning temperature and humidity show statistical significance but have minor impact on the radon concentration. The comparable estimates of the different models show a positive influence potential of $0.12-0.56 \%$ for the gradient of humidity and $1.0-2.6 \%$ for the gradient of temperature, each per change of unit within the gradient. Within the statistical models for the five locations, a special influence could be characterized for the bedroom. The temperature gradient shows a negative influence on the radon concentration in the room. This can be explained by the resident's behavior: when the temperature in the room rises, people tend to open the windows resulting in a decrease of the amount of radon. 


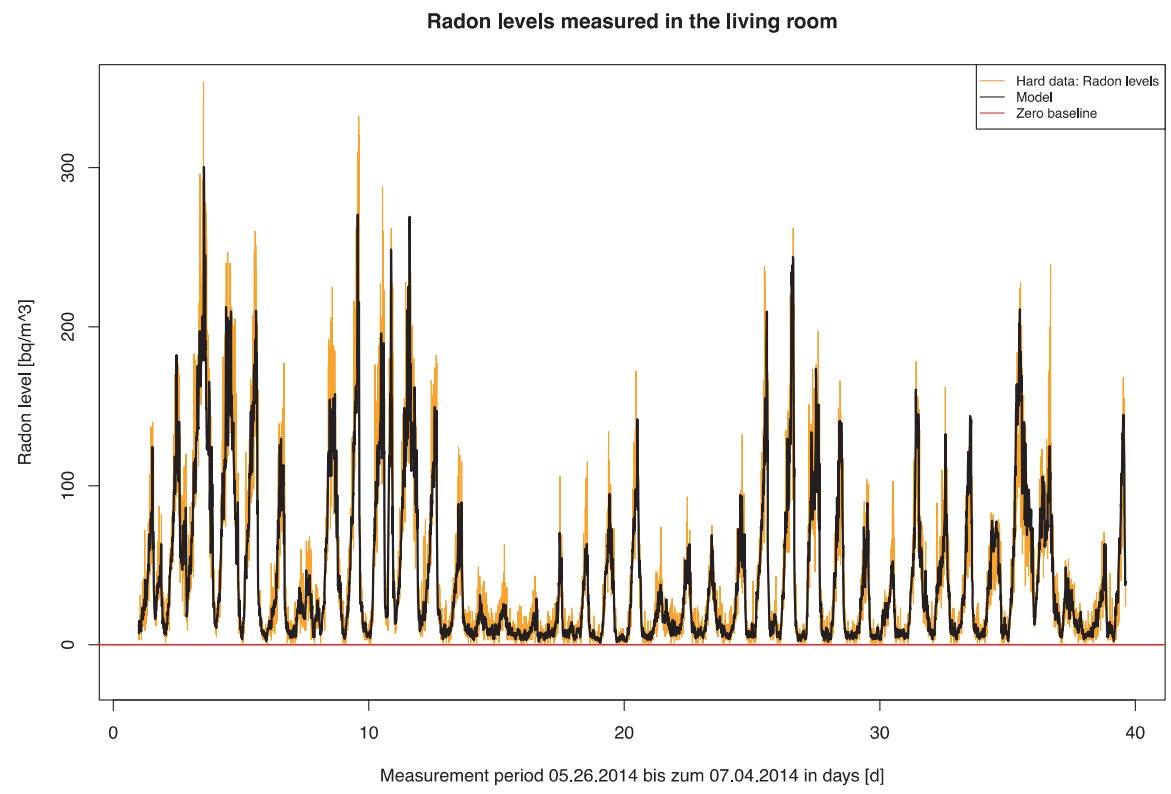

Fig. 5. Example model (living room; $\mathrm{r}^{2}=0.772$ ).

The gradient of ambient pressure was initially assumed to be a major influence factor on the radon concentration. The analysis of our models exhibits that in only two of the five rooms does the ambient pressure have an influence on the gas concentration. However, this influence was the strongest amongst the climate parameters with a range of positive influence of $8 \%$ to $14 \%$. The absence of this variable in the other models might be explained by a lack of sensitivity of the measuring device as the influence of the pressure is considerably highly reflected in the models through the autoregressive components of the different models.

The regression factor precipitation shows significant influence in most of the models. It appears with a consistent time lag and exhibits a positive effect on the gas concentration in a range of $2-4 \%$ in every room except the living room, where it shows no significance and a shorter time-lagged dependence. It is assumed that this phenomenon is caused by the resident: during rainfall, windows and doors often get closed, resulting in a rise of the amount of radon in the room.

Similarities as well as differences can be found within the significance and estimates of the dummy variables. The variable representing the usage of the room is present in four out of five location models and shows mean change of -5.4. The decrease in radon during occupation of a room seems to be the result of an effect on the movement of air in the building. Due to opening and closing the door, as well as leaving the door open while staying in a room, the radon concentration decreases if there exists a radon level gradient 
between adjacent rooms of the building. This gradient strongly depends on the usage of a room. Less frequent use of a room tends to create a higher concentration of radon because of undistorted build of the radon concentration. Another reason for the reduction of radon indicated by this variable is the fact that rooms often get ventilated after use, resulting in a steady air exchange and thereby limiting the saturation effect of radon.

A stronger influence is shown by the dummy variable indicating a highly increased ventilation of a room. This variable shows a high potential of changing the radon concentration of about $20-40 \%$ per ventilation event. The massive air exchanges of at least $10 \%$ during ten minutes have a strong influence on the airflow in the building. Air masses are drawn from the basement into the higher stories of the building. By closing the window, the air flow stops and then gathers in the higher levels of the building. As a result, higher radon concentrations can be measured.

The interpretation of the ventilation dummy variable has to be handled with care. Its influences depend highly on the ventilation behavior of the inhabitants, which might differ from room to room. Careful investigations are necessary for rooms that show very large estimates for this variable $(>50 \%)$. Rooms with only a few major ventilation events (typical example are basements) show implausible estimates because the regression model overestimates the importance of single event effects. Rooms that show an increased number of high value ventilation events are the bathrooms and bedrooms since they are used frequently and ventilated often. They are more likely to deliver an interpretable result. Regression models are sensitive to single events since these tend to receive high estimates representing the influence as well as statistical significance, but only because a small number of measurement points need to be fitted in the model. Therefore, estimates for single event variables are not representative for the influence of the variable.

The single event effect affects other variables too, such as the variable representing the wind speed. The device for data acquisition has a high threshold of $1 \mathrm{~km} / \mathrm{h}$ for measuring the wind speed. Furthermore, the massive influence of the mounting point has to be considered. The device easily ends up in a place of wind shadow during an acquisition period. A conclusive assumption on the influence of wind cannot be made using the current data, but further investigation is scheduled during a new data acquisition with other measurement tools. The statistic results are available upon request.

\section{Conclusion}

Our analysis of the regression models underlines that the main impact factor on the radon concentration in occupied houses is, as expected, the residents and their ventilation habits. Furthermore, meteorological parameters show influences even though these seem to be of minor importance. These parameters might even just show an indirect effect on the radon concentration in buildings, but rather affect the radon source itself. The latter is recognized via the autoregressive part of the regression model as the determining input factor for the radon concentration in the rooms. 
Based on our analysis, it is impossible to give advice on a suitable behavior for keeping the radon concentration low. Validations of the models on the basis of measurements in further houses are needed. Also, further investigations should try to define the influences on the radon source itself, which then will be validated with long term measurements and experiments in a fully controllable environment. This might give the ability to produce guidelines to keep the radon concentration low based on meteorological parameters and might even allow forecasts.

\section{Acknowledgments}

The presented method was part of the data analysis in a project funded by the German Federal Office for Radiation Protection, sponsoring number: BfS 3611 S 10016 Los 2.

\section{References}

1. Bundesministerium für Verkehr, Bau und Stadtentwicklung, Verordnung über energiesparenden Wärmeschutz und energiesparende Anlagentechnik bei Gebäuden (Energieeinsparverordnung EnEv, Berlin, 2009).

2. EURATOM, Basic safety standards, Office Journal of the European Union, 17, 01 (2014)

3. R: The R Project for Statistical Computing, http://www.r-project.org (2014).

4. Melissa A. Hardy, Regression with Dummy Variables (Sage Publication, Inc., California, 1993).

5. Andrew Gelman, Data Analysis Using Regression and Multilevel/Hierachical Models (Cambridge University Press, New York, 2007). 